\title{
Urinary catheter capable of repeated on-demand removal of infectious biofilms via active deformation
}

\author{
Vrad Levering $^{1,2 \xi}$, Changyong Cao ${ }^{1,3 \xi}$, Phanindhar Shivapooja ${ }^{1,2}$, Howard Levinson ${ }^{4}$, Xuanhe \\ Zhao ${ }^{1,3,5^{*}}$ and Gabriel P. López ${ }^{1,2,3^{*}}$ \\ ${ }^{1}$ Research Triangle Materials Research Science and Engineering Center, Duke University, \\ Durham, NC 27708, USA \\ 2 Department of Biomedical Engineering, Duke University, Durham, NC 27708, USA \\ ${ }^{3}$ Department of Mechanical Engineering and Materials Science, Duke University, Durham, NC \\ 27708, USA \\ ${ }^{4}$ Department of Surgery, Duke University School of Medicine, Durham, NC 27710, USA \\ ${ }^{5}$ Department of Mechanical Engineering, Massachusetts Institute of Technology, Boston, MA \\ 02139, USA
}

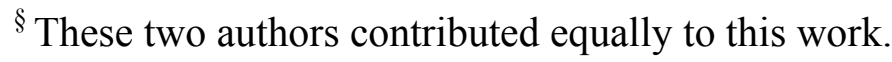

${ }^{*}$ To whom correspondence should be addressed. Email: gabriel.lopez@duke.edu or zhaox@mit.edu

\begin{abstract}
Biofilm removal from biomaterials is of fundamental importance, and is especially relevant when considering the problematic and deleterious impact of biofilm infections on the inner surfaces of urinary catheters. Catheter-associated urinary tract infections are the most common cause of hospital-acquired infections and there are over 30 million Foley urinary catheters used annually in the USA. In this paper, we present the design and optimization of urinary catheter prototypes capable of on-demand removal of biofilms from the inner luminal surface of catheters. The urinary catheters utilize 4 intra-wall inflation lumens that are pressure-actuated to generate
\end{abstract}


region-selective strains in the elastomeric urine lumen, and thereby remove overlying biofilms. A combination of finite-element modeling and prototype fabrication was used to optimize the catheter design to generate greater than $30 \%$ strain in the majority of the luminal surface when subjected to pressure. The catheter prototypes are able to remove greater than $80 \%$ of a mixed community biofilm of $P$. mirabilis and E. coli on-demand, and furthermore are able to remove the biofilm repeatedly. Additionally, experiments with the prototypes demonstrate that biofilm debonding can be achieved upon application of both tensile and compressive strains in the inner surface of the catheter. The fouling-release catheter offers the potential for a non-biologic, nonantibiotic method to remove biofilms and thereby for impacting the thus far intractable problem of catheter-associated infections.

Keywords: urinary catheter, biofilm removal, deformation, infection, finite element model.

\section{Introduction}

Infection associated with the use of urinary catheters is a pervasive and challenging issue in healthcare [1-6]. There are over 30 million urinary catheters used annually in the USA and catheter-associated urinary tract infections (CAUTIs) are the most common type of nosocomial infections, which account for $30-40 \%$ of all hospital infections and lead to over 50,000 deaths each year $[7,8]$. Microbes such as bacteria colonize the surface of urinary catheters very quickly and often form biofilms in the drainage lumen of catheters [9-15]. The formation of asymptomatic biofilms in urinary catheters promotes development of symptomatic CAUTIs [8], and nearly all patients that undergo catheterization for longer than 28 days will suffer some form

of infection [8]. In addition, CAUTIs also contribute to the alarming general increase in 
antibiotic resistance due to horizontal gene transfer between bacteria within biofilms, and the frequent use of antibiotics in their treatment [7, 16-18].

Current commericially marketed strategies, such as killing bacteria or delaying bacterial attachment $[10,19,20]$, to reduce infection induced by urinary catheters have been unsuccessful in the long-term prevention of biofilm formation which ultimately leads to CAUTIs $[7,8]$. Although recent research on techniques to prevent catheter infection such as bacterial interference [21] and phage delivery [22] show some promise, they are effective only against specific bacterial strains which prohibitively increases the difficulty of their implementation. Identification of the infecting strain(s) is not a typical clinical approach, and even more challenging is the huge variety of infectious microbes, both bacterial and fungal [10]. Indeed, even the most recently discovered new antibiotic is only effective on Gram positive bacteria [23]. Microtopography [4, 24, 25], permenantly attached silicone oils [26, 27], hydrogels [8, 28, 29], polymer brushes [30,31], and ultrasound [32] are other promising non-strain-specific strategies, but again they only delay biofilm formation for a short period and eventually a biofilm still forms. Moreover, the possible large cost to implement them are a hindrance to their routine implemention in clinical settings. Urinary catheters are commodity devices that cost approximately $\$ 10$ US; any additional technology that costs more than pennies to implement would face a high barrier to enter the urinary catheter market. Therefore, it is both practically important and fundamentally interesting to propose a new kind of antifouling or fouling release method to maintain catheters free of infection-promoting biofilms at minimal additional manufacturing cost.

With these considerations, we recently proposed an active control approach, adapted from our work on marine biofilms, which uses inflation-generated strain of the elastomeric 
substrate to debond overlying biofilms $[33,34]$. We found that increasing the strain in the substrate increases the energy release rate and thereby increases the driving force for debonding of the biofilm. We then used 3D printing to fabricate proof-of-concept (POC) urinary catheter prototypes that generate enough strain to succesfully debond and remove mature $P$. mirabilis biofilm from their interiors [34]. However, the POC prototypes left multiple questions of practical significance unresolved. The POC prototypes were less than $7 \mathrm{~cm}$ long and over $1.4 \mathrm{~cm}$ diameter, which is much shorter and stouter than the standard urinary catheter $(25-42 \mathrm{~cm}$ long and $5-10 \mathrm{~mm}$ in diameter). Additionally, the POC prototypes had only one intra-wall inflation lumen, resulting in straining and debonding of the biofilm from only part of the surface (about $35 \%$ of the intra-luminal perimeter). These limitations raised the following questions: does the active control approach work well for the length scales of a standard urinary catheter, in particular, with a small diameter catheter, e.g., around $6 \mathrm{~mm}$ ? Is it possible to remove biofilm from the full intra-luminal perimeter of the catheter? Although our pilot study demonstrated that strain applied on the substrate debonded a range of biofilms [33,34], would the technique work with a mixed community biofilm? Finally, we did not know whether the substrate strain would repeatedly debond biofilms (or if the approach would select from resilient biofilms) to allow long term use.

Here we present the design and a prototype of a urinary catheter capable of repeated ondemand biofilm removal. We hypothesized that adjusting the number and postion of intra-wall inflation lumens would allow inflation to generate sufficient tensile strain to debond biofilms over the majority of the internal lumen perimeter. We used successive rounds of finite element modeling to optimize the predicted strain of catheter cross sectional profiles to ensure the design fell within the fabrication capability of an industrial catheter manufacturer. We then constructed 
prototypes with clinically relevant dimensions using a combination of extrusion and 3D printed reversed-mold fabrication techniques. Different materials for the prototype catheter shaft were compared to determine the ideal operational parameters for clinicians to manually inflate our prototype. We further characterized the protoypes and compared their inflation performance against our finite element models. The prototype catheter, less than $7 \mathrm{~mm}$ in diameter (well within the range of sizes available for clinical use) with four intra-wall inflation lumens, was able to achieve substrate strain over most of the perimeter of the main drainage lumen, as well as along the full length of the device. We hypothesized that prototypes would debond a mixed community biofilm of E. coli and P. mirabilis, two of the most common bacteria found in CAUTIs, and we developed an artificial bladder flow system to grow mature biofilms inside the main drainage lumen of prototype catheters. Upon on-demand, inflation-generated actuation, the prototypes dramatically removed the vast majority of the biofilm along the full length of the catheter. After that first successful biofilm removal, we then regrew biofilm within the catheter and demonstrated that inflation-induced strain would indeed repeatedly remove biofilm in the catheter. Interestingly, upon disection of the catheters, we observed that areas that underwent compressive strain, as predicted by the finite element models, debonded biofilm similarly to areas that underwent tensile strain. In total, using currently avaliable manufacturing techniques from a catheter manufacturer, we developed a urinary catheter that allows the repeated and thorough removal of infectious biofilms from its interior; we are thus poised to impact the longstagnant urinary catheter technology market. 


\section{Materials and Methods}

Finite element modeling: Since catheters are relatively long compared to their cross-section dimensions, we simplified our design analysis to a plane-strain problem. In our analysis, the proposed catheter designs were modeled with the hybrid quadratic elements (CPE8MH) under plane-strain deformation using the software package, ABAQUS 6.12. Pressure was applied along the inner surfaces of the inflation lumens while a free boundary condition was used along the outer surface of the catheter to predict its radial displacements. Mesh density was determined by a convergence study and 10,441 CPE8MH elements were used for the whole model. A nonlinear solution method and geometric nonlinearity were adopted in the analysis. We used a $0.2 \mathrm{~mm}$ thick wall between the inflation lumen and the main lumen for models used for selecting the number of inflation lumens. Finite element models of the fabricated tubing used a $0.27 \mathrm{~mm}$ thick wall to reflect the actual dimensions achieved by the extrusion vendor. Three different materials are used for the catheters: 50 durometer silicone elastomer, 35 durometer silicone elastomer, and a more rigid sheath of 65 durometer silicone elastomer (all durometers defined per the type A scale), which were tested using a tensile tester (MSA Inc., USA) and fitted using the NeoHookean model with shear modulus of $0.69 \mathrm{MPa}, 0.52 \mathrm{MPa}$, and $2.44 \mathrm{MPa}$, respectively (Fig. S1). The strains along the internal surface of the drainage lumens and the average radial displacement along the outer surface were calculated by the finite element model for comparison against experimental results.

Preparation of prototypes: Vesta Inc. (Lanham, Maryland, USA) extruded silicone catheter shaft components according to our specifications using Dow Corning two-part, platinum-catalyzed Class VI silicone feedstock. Vesta Inc. varied the silicone feedstock to achieve 35 and 50 durometer multi-lumen silicone main shafts and the 65 durometer silicone sheath (all durometers 
defined per the type A scale). In instances where a sheath was used, we slip-fit the sheath over the main shaft using isopropyl alcohol. The inflation lumens were then sealed at each end of the main shaft using Sil-Poxy ${ }^{\circledR}$ silicone adhesive (Smooth-on Inc., USA). We then skived $2 \mathrm{~mm}$ long holes out of the outer walls of the inflation lumen approximately $1 \mathrm{~cm}$ from the designated hub end of the shaft. Hub manifolds were prepared by pouring silicone (Dragon Skin 0020®, Smooth-On Inc., USA) into a mold prepared by a 3D printer (Dimension SST 1200ES, with patterns generated using Solidworks 20131, Fig. S2). The inner diameter of hubs was approximately $0.5 \mathrm{~mm}$ greater than the shaft in order to create a manifold to allow simultaneous inflation of all four lumens. Once cured, the hubs were removed from the molds and then pierced and fit with a male touhy borst connector to be used for inflation. We fit the hubs over the designated hub end of the shaft and carefully glued the hub in place without occluding the skived holes in the inflation lumens, thus allowing simultaneous inflation of all four lumens via the touhy borst connector. Prototype performance was examined using optical video of on-end and side-views of inflation. We then analyzed still images from the video using ImageJ to characterize strain and dimensional parameters as a function of inflation pressure.

Bacteria strain and culture media: Proteus mirabilis 2573 (ATCC 49565) and Escherichia coli K12 (ATCC 29425) were thawed from frozen stock and cultivated overnight at $37^{\circ} \mathrm{C}$ on separate tryptone soya broth agar slants which were stored at $4^{\circ} \mathrm{C}$ and used for up to 2 weeks. The artificial urine media formulation was composed of urea $25 \mathrm{~g} / \mathrm{L}$, sodium chloride $4.6 \mathrm{~g} / \mathrm{L}$, potassium dihydrogen phosphate $2.8 \mathrm{~g} / \mathrm{L}$, disodium sulfate $2.3 \mathrm{~g} / \mathrm{L}$, potassium chloride $1.6 \mathrm{~g} / \mathrm{L}$, ammonium chloride $1.0 \mathrm{~g} / \mathrm{L}$, magnesium chloride hexahydrate $0.65 \mathrm{~g} / \mathrm{L}$, trisodium citrate dihydrate $0.65 \mathrm{~g} / \mathrm{L}$, calcium chloride $0.49 \mathrm{~g} / \mathrm{L}$, disodium oxalate $0.02 \mathrm{~g} / \mathrm{L}$, and gelatin $5.0 \mathrm{~g} / \mathrm{L}$ in deionized water and was prepared as described previously [34]. The artificial urine media was 
sterilized and then supplemented with $1.0 \mathrm{~g} / \mathrm{L}$ tryptone soya broth prepared separately to make the total artificial urine media (AUM) $[34,35]$. Colonies of $P$. mirabilis and E. coli were each inoculated into separate flasks of $75 \mathrm{mLs}$ of AUM and grown for 4 hours at $37^{\circ} \mathrm{C}$ on a shaker at $240 \mathrm{rpm}$.

Biofilm growth in catheter prototypes: We grew biofilms with a co-community of $P$. mirabilis and E. coli on the main drainage lumen of catheter prototypes using a continuous flow method as established by Stickler et al. [35], but modified to accommodate a manifold of four $50 \mathrm{~mL}$ artificial bladders in a vertical orientation (example artificial bladder shown Fig. S3). The distal (non-hub) tips of the prototype catheters were inserted through a pressure-fit seal in the bottom of the artificial bladders; they were inserted approximately $4 \mathrm{~cm}$ into the bladder to ensure the bladder would hold $30 \mathrm{~mL}$ before draining through the catheter. The catheter prototypes, artificial bladders, and all associated supply and drain tubing were sterilized and placed in a Class II biosafety cabinet. The bladders and prototypes were maintained at $37^{\circ} \mathrm{C}$ in a miniincubator. The bladders each held a $30 \mathrm{~mL}$ reservoir of infected media that would overflow into the distal tip of the catheter prototype and then drip-feed through the main drainage lumen of the prototypes as fresh media was added to the bladder. The system was primed with AUM, and then inoculated with 4 hour cultures of $5 \mathrm{~mL}$ of $P$. mirabilis and E. coli, each introduced into the artificial bladder. The bacteria were left for 1 hour to allow attachment and infection of the bladders and catheters. The model was then run continuously at a flow rate of $0.5 \mathrm{~mL} \min ^{-1}$ supplied via peristaltic pumping until the desired time point when a thick biofilm was visible through the walls of the prototype, or a system blockage occurred. All biofilm growth was conducted in a sterile biosafety cabinet. The sterility of the artificial bladder growth system was 
confirmed by control runs without bacterial inoculation; no deposition was visually observed and microscopic examination confirmed no biofilm was formed on control samples.

Actuation testing: For the samples undergoing only one round of biofilm removal, the prototypes were gently removed from the artificial bladders and kept covered in a hydrated state. The samples were suspended vertically, and artificial urine media was introduced into the upper end at a flow rate of $4 \mathrm{~mL} \mathrm{~min}^{-1}$ for 1 minute (Fig. S3). Samples designated for inflation were rapidly inflated to a pressure of $80 \mathrm{kPa}$ and then deflated 10 times at $0.6 \mathrm{~s}^{-1}$ to achieve $35 \%$ average strain, each inflate/deflate cycle taking less than one second, approximately 20 seconds into the 1 minute rinse. Inflation was conducted hydraulically using a syringe-delivered, predetermined volume of water. Prototype samples were weighed before biofilm growth, before rinse, and after the rinse in order to assess the weight of biofilm grown and removed. The effluent from each sample's rinse was also collected. We centrifuged the effluent, removed the liquid supernatant, and weighed the remaining biofilm as another measure of biofilm removal. Samples were then dissected into tip, top, middle and bottom sections. $1 \mathrm{~mm}$ thick sections for cross-sectional views of the main lumen and $1 \mathrm{~cm}$ long sections that were filleted in half for longitudinal views of the main lumen were sliced from the top, middle, and bottom sections. Those sections, in addition to cross sectional views of the tip, were then optically photographed. Image analysis to quantify the biofilm occlusion of the luminal cross-sectional area was conducted using representative images of unstained cross-sections and ImageJ version $1.49 \mathrm{v}$ (rsbweb.nih.gov/ij/). Briefly, the image contrast was increased by $0.3 \%$ to highlight the biofilm, and the image was rendered as a binary image to show distinct areas with and without biofilm. ImageJ's area fraction measurement function was then applied to the luminal cross-sectional area. Additional pieces from the top, middle, and bottom were stained with $0.01 \%$ crystal violet 
for 10 minutes and rinsed 2 times with DI water before similar slicing for cross sectional and longitudinal views. Representative longitudinal, crystal violet stained samples were carefully cut to excise the main lumen from the catheter shaft to allow flattened views of the biofilm coverage of the main lumen. Stained sections were also optically photographed, and selected sections were examined on the phase microscope at 10x magnification.

Fresh prototype catheter samples were fabricated to undergo two rounds of biofilm removal. The co-community biofilm of $P$. mirabilis and E. coli was grown on the main drainage lumen of catheter prototypes using the same continuous flow method described above. We utilized inflation actuation as described above to remove the biofilm from all samples once the biofilm formed. We then replaced the consumed supply of AUM with a fresh supply of AUM, and emptied the drainage collection flask before re-starting the peristaltic pump at the same flow rate of $0.5 \mathrm{~mL} \mathrm{~min}^{-1}$. Once the co-community biofilm regrew (after approximately 24 hours), we stopped the flow. The artificial bladders and the catheter samples were carefully removed from the flow loop and all catheters were rinsed with AUM supplied into the artificial bladder at a flow rate of $4 \mathrm{~mL} \mathrm{~min}^{-1}$ for 1 minute. Samples designated for inflation were rapidly inflated to a pressure of $100 \mathrm{kPa}$ and deflated 10 times to achieve $40 \%$ strain approximately 20 seconds into the 1 minute rinse. The effluent from each sample's rinse was collected and samples were then dissected as described above. Image analysis to quantify the biofilm occlusion of the luminal cross-sectional area was conducted as described above.

Statistical Analysis: Statistical comparisons were conducted using GraphPad Prism 5 (USA). Group means were compared by two-tailed, unpaired $t$-tests with Welch's correction to account for potentially unequal variances. “*” denotes $P<0.05$, “**” denotes $P<0.01$ and “***” 
denotes $P<0.001$ where shown in figures. Data are presented as mean $+/$ - standard deviation in bar and line graphs.

\section{Results and Discussion}

\section{Shaft design}

As depicted in Fig. 1, we have proposed a simple but new concept for a urinary catheter prototype capable of releasing biofilms by means of active actuation of elastomers [34]. The design is based on equipping the catheter with inflation lumens between the inner main lumen and outer catheter wall (Fig. 1a). After infectious biofilms form on the surface of the main drainage lumen (Fig. 1b), we pneumatically or hydraulically actuate the inflation lumens to a controlled level of strain for multiple inflate/deflate cycles (Fig. 1c). As a consequence, the biofilm is debonded from the surface of the main lumen and then easily removed by a minimal flow of liquid (e.g., urine generated by a patient, Fig. 1d), thereby clearing the urine drainage lumen for continued use.

In our previous work, we found that active surface deformation effectively detaches mature crystalline urinary biofilms from flat and curved surfaces of silicone elastomers. We also showed that both the strain rate and strain level generated by actuation has a significant influence on biofilm debonding [34]. The biofilm debonds once the energy release rate exceeds the adhesion strength between the biofilm and the substrate. Since this validation of the general concept for biofilm release, we have focused, and report herein, the development of designs for a new generation of urinary catheters with the ability of on-demand biofilm release. The small cross section of the catheter and the limitations of the manufacturer's capabilities required us to 
carefully optimize the design, while the design process itself revealed new and fundamental insights about inflation and biofilm debonding performance in constrained regions.

In order to actuate the maximum area of the drainage lumen's surface within the catheter, we designed the inflation lumens to underlie as much of the perimeter as possible. We used the finite element models to predict inflation performance and the resultant strains in the wall of the main lumen. We first designed a two-inflation-lumen catheter, in which each inflation lumen takes up almost half of the perimeter of the catheter (see Fig. 2a). The finite element model clearly demonstrated that, after an initial increase of the surface strain on the surface of main drainage lumen, as inflation pressure increased, the surface strain stops increasing at $\sim 15 \%$ due to the interfering contact of the two walls in the confined space of the drainage lumen (Fig. 2c). From our previous study, we know that the biofilm debonds once the energy release rate, $G$, exceeds the adhesion strength between the biofilm and the substrate due to applied strain, and $G \propto \mu_{f} \varepsilon^{2} H$ (where $\mu_{f}$ is the storage modulus of the biofilm, $\varepsilon$ is the applied strain in the substrate, and $H$ is the biofilm thickness) [33]. We found that the majority of the biofilm debonds once the applied strain in the substrate reaches a "critical" value, $\varepsilon_{c}$ [33]. For instance, in mucoid biofilms such as E. coli, the majority of the bioflm debonds at a critical strain of $15 \%$ (although critical strain can vary depending upon biofilm thickness [33] and substrate modulus [36]); and in crystalline biofilms such as $P$. mirabilis, the critical strain is approximately $25 \%$ [34]. We desired to exceed $30 \%$ strain as a conservative design goal [34].

We considered programmed, sequential inflation of the two-inflation-lumen design to achieve the desired critical strains, but this caused large distortion of the cross-section outer diameter (Fig. S4). Therefore, to limit interference between inflated lumens, we reduced the perimeter length of the individual inflation lumens while increasing the number of inflation 
lumens to four (Fig. 2d). Using finite element models (Fig. 2e), we predicted that the strains along the internal surface of the drainage lumen would reach greater than the desired $30 \%$ strain at a pressure load of approximate $70 \mathrm{kPa}$ (assuming silicone with a shear modulus of $0.68 \mathrm{MPa}$ ). Health care providers easily achieve $70 \mathrm{kPa}$ using common hospital syringes, so we moved forward with a four-inflation-lumen design. It was also readily apparent that the luminal surface overlying the walls between the inflation lumens could not be inflated during the actuation process. However, high compressive strains in these regions were expected due to the inflation on either side of these regions (i.e. inflation on either side "squeezes" the in-between wall area) (Fig. 2e). We thus hypothesized that the biofilm overlying the wall area would also debond from the substrate surface due to the large mismatched strain introduced by compression.

\section{Catheter fabrication}

We contracted with Vesta Inc. (Lanham, Maryland, USA) (which routinely extruded silicones for the medical industry) to produce a series of iterations of the multi-lumen catheter shafts for our prototypes. Based on manufacturing limitations in fabricating high-aspect-ratio inflation lumens, we optimized the shape of cross sections of the inflation lumens for manufacturing using both finite element simulations and laboratory experiments with fabricated prototypes (see Section S5, Fig. S5, and Fig. S6). We first modeled, and then fabricated, catheter prototypes using a 50 durometer silicone (Dow Corning two-part, platinum-catalyzed Class VI silicone feedstock; 50 durometer extrusion from Vesta Inc.), but found the inflation required a higher inflation pressure than was manually achievable (Fig. S5). We repeated the finite element modeling and fabrication using a "softer" 35 durometer silicone and achieved sufficient inflation at a reasonable $70 \mathrm{kPa}$ (Fig. S6). The 35 durometer catheter prototypes, however, exhibited excessive outward inflation (change in external diameter) (Fig. S6b). Therefore, we finally added a thin-walled, higher- 
modulus (65 durometer) "sheath" to the outside of the catheter to constrain the deformation of the outer surface (Fig. 3b).

We further employed finite element models to estimate the strains under different inflation pressures of the cross-section of the optimized, final version of the urinary catheter shaft, which has four inflation lumens and was made of low modulus silicone (35 durometer) and constrained with a high-modulus (65 durometer) silicone sheath. Fig. 3c shows the average strains obtained as a function of the applied hydraulic pressure. The elastomer for the sheath was assumed to be a Neo-Hookean material with a shear modulus of $2.44 \mathrm{MPa}$ (Fig. S1b). Simulation results confirmed that the inflated wall easily achieved substrate strains sufficient to debond crystalline biofilms (e.g., greater than 30\% strain) over most of the surface (Fig. 3c). As shown in Fig. 3d, the change in the outer radius of the shaft at higher pressure was dramatically reduced with the added sheath. We experimentally actuated the catheter with sheath using colored water and verified that the numerical results agree well with experimental data in the relevant range (Fig. 3c and d) and exhibited similar appearance during the inflation process (Video S1 and Fig. S7). Fig. 3b shows the deformation profile of the four inflation lumen catheter, which is similar to the profile predicted by the strain contour plot at $80 \mathrm{kPa}$ as shown in Fig. 3a.

\section{Biofilm debonding}

We next experimentally demonstrated the efficacy of the new catheter in debonding a mixed community biofilm of $P$. mirabilis and E. coli from the main drainage lumen surface of the catheter prototype in an in vitro biofilm model. E. coli is present in up to $90 \%$ of diagnosed urinary tract infections, and $P$. mirabilis is another frequent infecting bacterium that can accumulate in thickness sufficiently to block the urinary catheter causing trauma, leakage, 
polynephritis, and septicemia while overall being very difficult to treat $[2,10]$. We chose $P$. mirabilis and E. coli to represent a challenging-to-remove and yet typical mixed community biofilm. Additionally, the two species have been shown to be non-interfering in a urinary catheter model, so we hypothesized that they would form a robust mixed-community biofilm $[2,10]$. We employed an artificial bladder biofilm growth model modified to fit our prototypes. The model fed infected artificial urine downward through prototypes (Fig. S3) at a rate of $0.5 \mathrm{~mL} \mathrm{~min}^{-1}$, and after approximately 30 hours achieved uniform biofilm distribution around the perimeter and down the length of the main lumen (see Fig. 4a for uninflated control sample). Our previous experiment with a similar biofilm growth model with only $P$. mirabilis had required approximately 42 hours to achieve a mature biofilm [34], and after accounting for the differences in geometry and dimensions it appeared that the two bacterial strains exhibited a mild synergistic influence on biofilm growth.

Once a mature biofilm was clearly visible covering the interior of the catheter, we gently removed the catheters from the artificial bladders and mounted them vertically for rinsing and testing (see Fig. S3c). Each catheter was rinsed with artificial urine media supplied at $4 \mathrm{~mL} \mathrm{~min}^{-1}$ for $1 \mathrm{~min}$. Catheters that we designated for inflation/actuation, were rapidly inflated to $80 \mathrm{kPa}$ and deflated 10 times, achieving an average of approximately $35 \%$ strain, at $20 \mathrm{~s}$ into the rinse (see supplemental videos). The debonding of the biofilm due to the actuation and the subsequent removal of the biofilm in the effluent was visually observed through the walls of the catheter. In cases where the catheter was almost clogged with biofilm, the debonded biofilm would flow downward and then re-clog at the hub. We realized that imperfections in the hub region were creating a choke point and thus inserted a plastic tube into the main lumen to shunt past the hub, which allowed the biofilm to flow out from the catheter in subsequent runs. We collected the 
effluent, centrifuged it (average relative centrifugal force of 716,5 minute duration, $22{ }^{\circ} \mathrm{C}$ ), removed the supernatant, and then weighed the remaining biofilm in order to quantify the biofilm detachment (biofilm mass removed normalized to the biofilm mass grown) for a particular run.

Finally the catheter was removed and sectioned to facilitate observation of the biofilm on the main drainage lumen surface. Sections from the top, middle, and bottom of the catheter shaft were also stained with $0.1 \%$ crystal violet to enhance biofilm visualization. Fig. 4 shows representative optical images of the cross sections of control urinary catheter shaft with mixed community $P$. mirabilis and $E$. coli biofilm intact on the main lumen of a control vs. an actuated catheter. As shown in the representative images, the majority of the biofilm accumulated in the main lumen was clearly removed by inflation. We statistically analyzed the normalized biofilm mass removed and confirmed that the inflation removed a large fraction $(\approx 80 \%)$ of $P$. mirabilis and E. coli biofilm mass ( $<0.005$ for $\mathrm{N}=3$ replicates). Representative unstained cross sections from each catheter were also analyzed for the fraction of luminal cross sectional area occluded by biofilm, and the image analysis confirmed that little biofilm remained in the lumen of inflated samples ( $\mathrm{p}<0.01$, see Fig. $4 \mathrm{c})$.

We visually and microscopically observed that the biofilm exhibited a predominantly crystalline composition as is typical with mixed community biofilms containing ureaseproducing strains such as $P$. mirabilis $[34,37]$. In order to analyze the mechanical properties of the co-biofilm, we grew the mixed community biofilm on flat silicone samples as previously described [34]. We tested the complex visco-elastic modulus of the biofilms using an AR G-2 Rheometer and found that the mixed community biofilms demonstrated properties similar to our previous results for single-strain $P$. mirabilis biofilms [34]. The mixed community biofilms of $P$. 
mirabilis and E. coli were predominantly elastic with a storage modulus, $G^{\prime}$, of $\sim 2.5 \times 10^{4} \mathrm{~Pa}$ and loss modulus, $G^{\prime \prime}$, of $\sim 3.9 \times 10^{3}$ Pa for the scanned frequencies (See Section S8 and Fig. S8). We also carefully tested the adhesion strength of the biofilm based on a modified scratch test (Fig. S9) [38] and found that the co-biofilm exhibited an adhesion strength of approximately $\sim 8 \mathrm{~J}$ $\mathrm{m}^{-2}$.

\section{Repeated biofilm debonding}

To assess the performance of the catheter in repetitive debonding of the biofilm for long term use, we regrew biofilm using the artificial bladder system for $24 \mathrm{~h}$ after initially debonding the biofilm from all of the sample catheters after $\approx 30$ hours of biofilm growth. We chose to leave the catheter prototypes "in situ" in the artificial bladders during the rinse and debonding steps to more closely simulate clinical conditions. Artificial urine media accumulated in the artificial bladders before flowing into the distal tip of the catheter, instead of being fed directly into the distal tip. Catheters designated for inflation after the second round of biofilm growth were rapidly inflated to $100 \mathrm{kPa}$ (approximately 40\% strain) and deflated 10 times approximately 20 seconds into the rinse. We again observed biofilm debonding from the main drainage lumen upon inflation actuation, and collected and weighed biofilm mass in the rinse effluent. Fig. 5c describes the performance of the second run of debonding after re-growing the biofilm; actuation again removed the majority of the mixed community biofilm $(83.6 \pm 6.2 \% \mathrm{~N}=4)$ at a statistically significant level $(\mathrm{p}<0.001)$. The prototypes were removed, sectioned, and optically imaged. Fig. $5 \mathrm{a}$ and $\mathbf{5 b}$ show the representative optical images from cross sections that were crystal violet stained to enhance visualization. Control samples show thick biofilm coverage and inflated samples confirm substantial biofilm removal. We again used representative unstained cross sections from each catheter to assess the fraction of luminal cross sectional area occluded by 
biofilm, and confirmed that little biofilm remained in the lumen of inflated samples $(\approx 1.9 \%$, $\mathrm{p}<0.05$, see Fig. $5 \mathrm{c})$.

Fig. 6 shows optical images of cross sections along the length of three representative urinary catheters' shafts; a control, a catheter that underwent one round of biofilm debonding, and a catheter that underwent two rounds of biofilm debonding. We show the unstained cross sections since the distal tips (the end without a hub) were not stained, but the stained cross sections had similar results. Biofilm removal clearly occurs along the length of the catheter, thereby confirming our hypothesis that the intra-wall actuation would work along the length of the catheter. Additionally, the second round of biofilm removal appeared to be just as successful at removing biofilm as the first actuation. If we compare the fraction of biofilm removed for the first round of biofilm removal against the second, we find no statistical difference between the data sets $(\mathrm{N}=3)$.

\section{Compressive strain of the substrate to debond biofilms}

Previous studies have shown in several milieus that tensile strain to the substrate can debond overlying biofilm from the substrate [33, 34]. However, the shafts of these prototype catheters represent the first opportunity to evaluate the impact of compressive strain on biofilm debonding. Fig. $7 \mathrm{a}$ shows the strain predicted by finite element models to have occurred in a catheter inflated to $100 \mathrm{kPa}$, and maps the absolute value of the strain onto the surface of the catheter after deflation. The area of the luminal surface overlying the wall between intra-wall inflation lumens (i.e., the connecting wall) does not undergo tensile strain, but does undergo a significant amount of compressive strain. Interestingly, the area of the luminal surface that undergoes the least strain is the very edge of the intra-wall inflation lumen, where the strain transitions from tensile to compressive and presents as an area of low absolute strain. When we examined 
longitudinal sections of catheters that had undergone actuation to debond biofilm (see representative section Fig. 7b), it appeared that areas of the luminal surface that we predicted had undergone compressive strain still had debonded the majority of the biofilm. We carefully excised the luminal surface from the rest of the catheter shaft in representative samples and took optical images (Fig. 7c) and microscope images (Fig. 7d) and confirmed that the biofilm was removed in areas of high compressive strain, and residual biofilm was at the predicted edge of the inflation area where we predicted low strain values.

\section{Conclusion}

Active surface deformation is an efficient but simple method for detaching biofilm from a silicone substrate. We demonstrated a prototype of a multi-inflation-lumen urinary catheter with the ability to debond biofilms from the previously-inaccessible main drainage lumen to keep its functionality. With the guidance of finite element analysis and experimental testing, we developed a design of an extrudable catheter shaft with four intra-wall inflation lumens that apply sufficient strains to debond biofilm around the majority of the intra-luminal perimeter. We further characterized the performance of the catheters by growing mixed community biofilms of E. coli and $P$. mirabilis on the main drainage lumen of the prototypes. The catheter prototypes were a clinically relevant dimension and removed the biofilm on-demand repeatedly. The design could be further optimized in the future with the use in conjunction of other methods to reduce the adhesion of the main luminal surface [4, 24-27]. The ability to repeatedly remove biofilm suggests the real-world applicability of our catheter design for use in the clinic where CAUTIs remain a challenging and pervasive problem. Upon close inspection of the interior of the catheter, we also discovered the first demonstration of debonding of the overlying biofilm due to 
compressive strain in the substrate. The new prototype fouling-release catheter was made using readily-available techniques suitable to cost-effective, large-scale medical device manufacture.

Our design offers a potential non-antibiotic, non-biologic approach to controlling biofilms and thereby reducing the persistent burden of CAUTIs.

\section{Acknowledgements}

This work was financially supported by the NSF's Research Triangle Materials Research Science and Engineering Center (DMR-1121107), the Office of Naval Research (N0014-13-1-0828), and the Duke-Coulter Translational Partnership Grant Program. Catheter image from Fig. 1 licensed from Shutterstock Inc.

\section{References}

[1] Kambal C, Chance J, Cope S, Beck J. Catheter-associated UTIs in patients after major gynaecological surgery. Prof Nurse. 2004;19:515-8.

[2] Jacobsen SM, Stickler DJ, Mobley HL, Shirtliff ME. Complicated catheter-associated urinary tract infections due to Escherichia coli and Proteus mirabilis. Clin Microbiol Rev. 2008;21:2659.

[3] Wang R, Neoh KG, Shi Z, Kang ET, Tambyah PA, Chiong E. Inhibition of Escherichia coli and Proteus mirabilis adhesion and biofilm formation on medical grade silicone surface. Biotechnol Bioeng. 2012;109:336-45.

[4] Reddy ST, Chung KK, McDaniel CJ, Darouiche RO, Landman J, Brennan AB.

Micropatterned surfaces for reducing the risk of catheter-associated urinary tract infection: an in vitro study on the effect of sharklet micropatterned surfaces to inhibit bacterial colonization and migration of uropathogenic Escherichia coli. J Endourol. 2011;25:1547-52.

[5] Sabbuba N, Hughes G, Stickler DJ. The migration of Proteus mirabilis and other urinary tract pathogens over Foley catheters. BJU Int. 2002;89:55-60.

[6] Stickler D, Young R, Jones G, Sabbuba N, Morris N. Why are Foley catheters so vulnerable to encrustation and blockage by crystalline bacterial biofilm? Urol Res. 2003;31:306-11.

[7] Hooton TM, Bradley SF, Cardenas DD, Colgan R, Geerlings SE, Rice JC, et al. Diagnosis, prevention, and treatment of catheter-associated urinary tract infection in adults: 2009

International Clinical Practice Guidelines from the Infectious Diseases Society of America. Clin Infect Dis. 2010;50:625-63.

[8] Siddiq DM, Darouiche RO. New strategies to prevent catheter-associated urinary tract infections. Nat Rev Urol. 2012;9:305-14.

[9] Mulla SA, Revdiwala S. Assessment of biofilm formation in device-associated clinical bacterial isolates in a tertiary level hospital. Indian J Pathol Microbiol. 2011;54:561-4. 
[10] Williams GJ, Stickler DJ. Effect of triclosan on the formation of crystalline biofilms by mixed communities of urinary tract pathogens on urinary catheters. J Med Microbiol.

2008;57:1135-40.

[11] Hall-Stoodley L, Costerton JW, Stoodley P. Bacterial biofilms: from the natural environment to infectious diseases. Nat Rev Microbiol. 2004;2:95-108.

[12] Ahimou F, Semmens MJ, Novak PJ, Haugstad G. Biofilm cohesiveness measurement using a novel atomic force microscopy methodology. Appl Environ Microbiol. 2007;73:2897-904.

[13] Pavithra D, Doble M. Biofilm formation, bacterial adhesion and host response on polymeric implants--issues and prevention. Biomed Mater. 2008;3:034003.

[14] Costerton JW, Montanaro L, Arciola CR. Biofilm in implant infections: its production and regulation. Int J Artif Organs. 2005;28:1062-8.

[15] Donlan RM. Biofilm formation: a clinically relevant microbiological process. Clin Infect Dis. 2001;33:1387-92.

[16] Campoccia D, Montanaro L, Arciola CR. A review of the clinical implications of antiinfective biomaterials and infection-resistant surfaces. Biomaterials. 2013;34:8018-29.

[17] Davey P, Brown E, Fenelon L, Finch R, Gould I, Holmes A, et al. Systematic review of antimicrobial drug prescribing in hospitals. Emerg Infect Dis. 2006;12:211-6.

[18] Dellit TH, Owens RC, McGowan JE, Jr., Gerding DN, Weinstein RA, Burke JP, et al. Infectious Diseases Society of America and the Society for Healthcare Epidemiology of America guidelines for developing an institutional program to enhance antimicrobial stewardship. Clin Infect Dis. 2007;44:159-77.

[19] Epstein AK, Hochbaum AI, Kim P, Aizenberg J. Control of bacterial biofilm growth on surfaces by nanostructural mechanics and geometry. Nanotechnology. 2011;22:494007. [20] Hazan Z, Zumeris J, Jacob H, Raskin H, Kratysh G, Vishnia M, et al. Effective prevention of microbial biofilm formation on medical devices by low-energy surface acoustic waves.

Antimicrobial agents and chemotherapy. 2006;50:4144-52.

[21] Flores-Mireles AL, Pinkner JS, Caparon MG, Hultgren SJ. EbpA vaccine antibodies block binding of Enterococcus faecalis to fibrinogen to prevent catheter-associated bladder infection in mice. Science translational medicine. 2014;6:254ra127.

[22] Kelly D, McAuliffe O, Ross RP, Coffey A. Prevention of Staphylococcus aureus biofilm formation and reduction in established biofilm density using a combination of phage $\mathrm{K}$ and modified derivatives. Letters in applied microbiology. 2012;54:286-91.

[23] Ling LL, Schneider T, Peoples AJ, Spoering AL, Engels I, Conlon BP, et al. A new antibiotic kills pathogens without detectable resistance. Nature. 2015.

[24] Chung KK, Schumacher JF, Sampson EM, Burne RA, Antonelli PJ, Brennana AB. Impact of engineered surface microtopography on biofilm formation of Staphylococcus aureus.

Biointerphases. 2007;2:89-94.

[25] Epstein AK, Hong D, Kim P, Aizenberg J. Biofilm attachment reduction on bioinspired, dynamic, micro-wrinkling surfaces. New J Phys. 2013;15.

[26] Leslie DC, Waterhouse A, Berthet JB, Valentin TM, Watters AL, Jain A, et al. A

bioinspired omniphobic surface coating on medical devices prevents thrombosis and biofouling. Nature biotechnology. 2014;32:1134-40.

[27] Wong TS, Kang SH, Tang SK, Smythe EJ, Hatton BD, Grinthal A, et al. Bioinspired selfrepairing slippery surfaces with pressure-stable omniphobicity. Nature. 2011;477:443-7.

[28] Morris NS, Stickler DJ, Winters C. Which indwelling urethral catheters resist encrustation by Proteus mirabilis biofilms? Br J Urol. 1997;80:58-63. 
[29] Stickler DJ. Bacterial biofilms in patients with indwelling urinary catheters. Nat Clin Pract Urol. 2008;5:598-608.

[30] Shivapooja P, Ista LK, Canavan HE, Lopez GP. ARGET-ATRP synthesis and characterization of PNIPAAm brushes for quantitative cell detachment studies. Biointerphases. 2012;7:32.

[31] Yang WJ, Neoh KG, Kang ET, Teo SLM, Rittschof D. Polymer brush coatings for combating marine biofouling. Prog Polym Sci. 2014;39:1017-42.

[32] Dror N, Mandel M, Hazan Z, Lavie G. Advances in microbial biofilm prevention on indwelling medical devices with emphasis on usage of acoustic energy. Sensors (Basel). 2009;9:2538-54.

[33] Shivapooja P, Wang Q, Orihuela B, Rittschof D, Lopez GP, Zhao X. Bioinspired surfaces with dynamic topography for active control of biofouling. Adv Mater. 2013;25:1430-4.

[34] Levering V, Wang Q, Shivapooja P, Zhao X, Lopez GP. Soft robotic concepts in catheter design: an on-demand fouling-release urinary catheter. Advanced healthcare materials. 2014;3:1588-96.

[35] Stickler DJ, Morris NS, Winters C. Simple physical model to study formation and physiology of biofilms on urethral catheters. Methods Enzymol. 1999;310:494-501.

[36] Phanindhar Shivapooja QW, Lizzy M. Szott, Beatriz Orihuela, Daniel Rittschof, Gabriel P. López and Xuanhe Zhao. Dynamic surface deformation of silicone elastomers for management of marine bofouling: laboratory and field studies using pneumatic actuation. Biofouling. In press 2015.

[37] Macleod SM, Stickler DJ. Species interactions in mixed-community crystalline biofilms on urinary catheters. J Med Microbiol. 2007;56:1549-57.

[38] Chen MJ, Zhang Z, Bott TR. Direct measurement of the adhesive strength of biofilms in pipes by micromanipulation. Biotechnol Tech. 1998;12:875-80.

[39] Pavlovsky L, Sturtevant RA, Younger JG, Solomon MJ. Effects of Temperature on the Morphological, Polymeric, and Mechanical Properties of Staphylococcus epidermidis Bacterial Biofilms. Langmuir. 2015;31:2036-42.

[40] Chen MJ, Zhang Z, Bott TR. Effects of operating conditions on the adhesive strength of Pseudomonas fluorescens biofilms in tubes. Colloids and Surfaces B: Biointerfaces. 2005;43:6171.

[41] Garrett T, Bhakoo M, Zhang Z. Characterisation of bacterial adhesion and removal in a flow chamber by micromanipulation measurements. Biotechnol Lett. 2008;30:427-33. [42] Meyers M, Chawla K. Mechanical Behavior of Materials, 1999. Prentice-Hall, Upper Saddle River. 


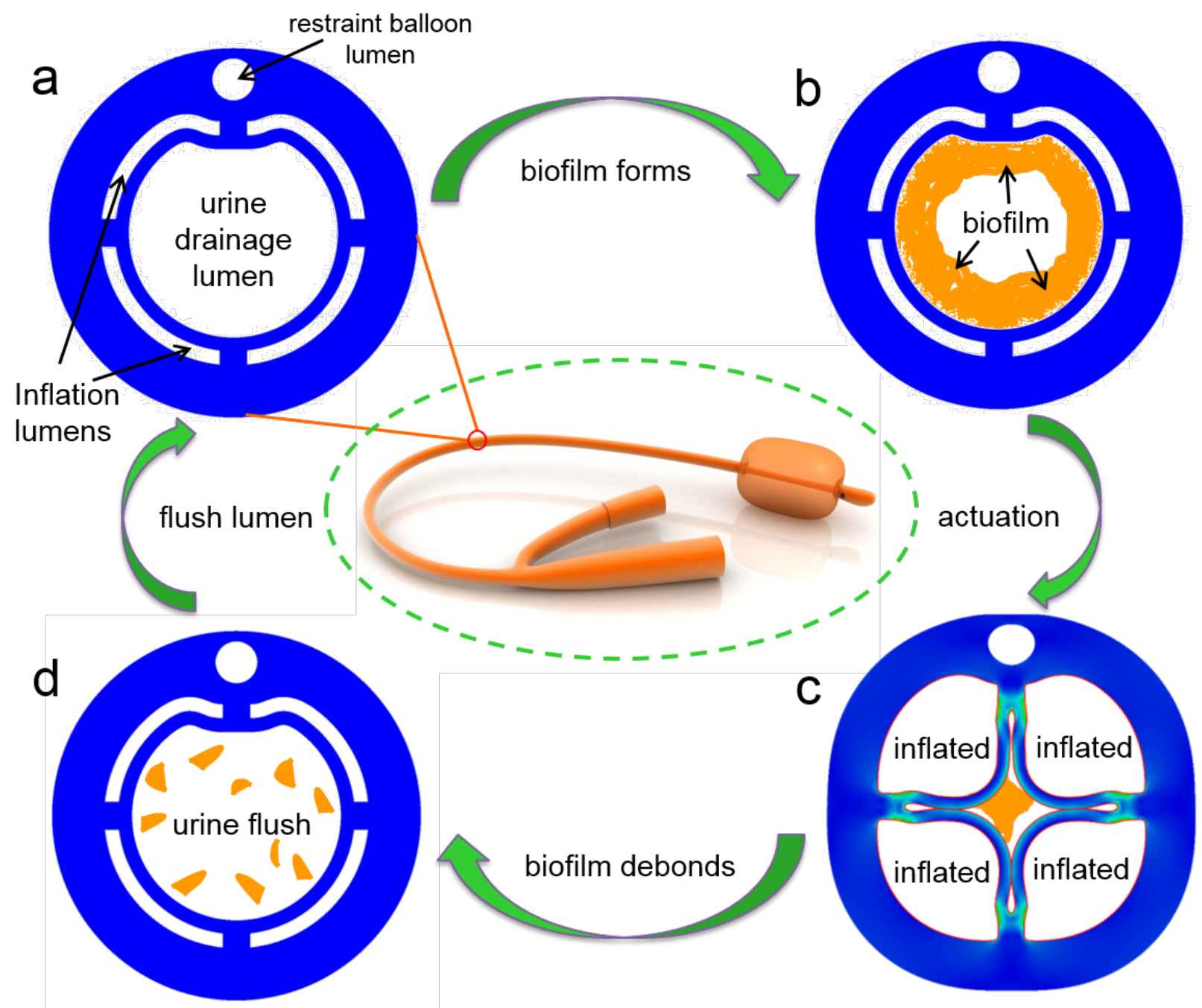

Fig. 1. Schematic of a urinary catheter capable of on-demand removal of infectious biofilms via active deformation. (a) Cross-section of the designed urinary catheter shaft with intra-wall inflation lumens. (b) Biofilm forms on the surface of urine drainage lumen after 1-2 days. (c) Actuation of inflation lumens by pumping air or water to generate large mismatched strains between biofilm and the surface of main lumen to debond the biofilm from the urine drainage lumen. (d) The detached biofilm is removed by the flow of urine once the inflation lumens are 
deflated. Therefore, the catheter can be maintained free of mature biofilms for long-term use and thereby may reduce the risk of catheter-associated urinary tract infections. 

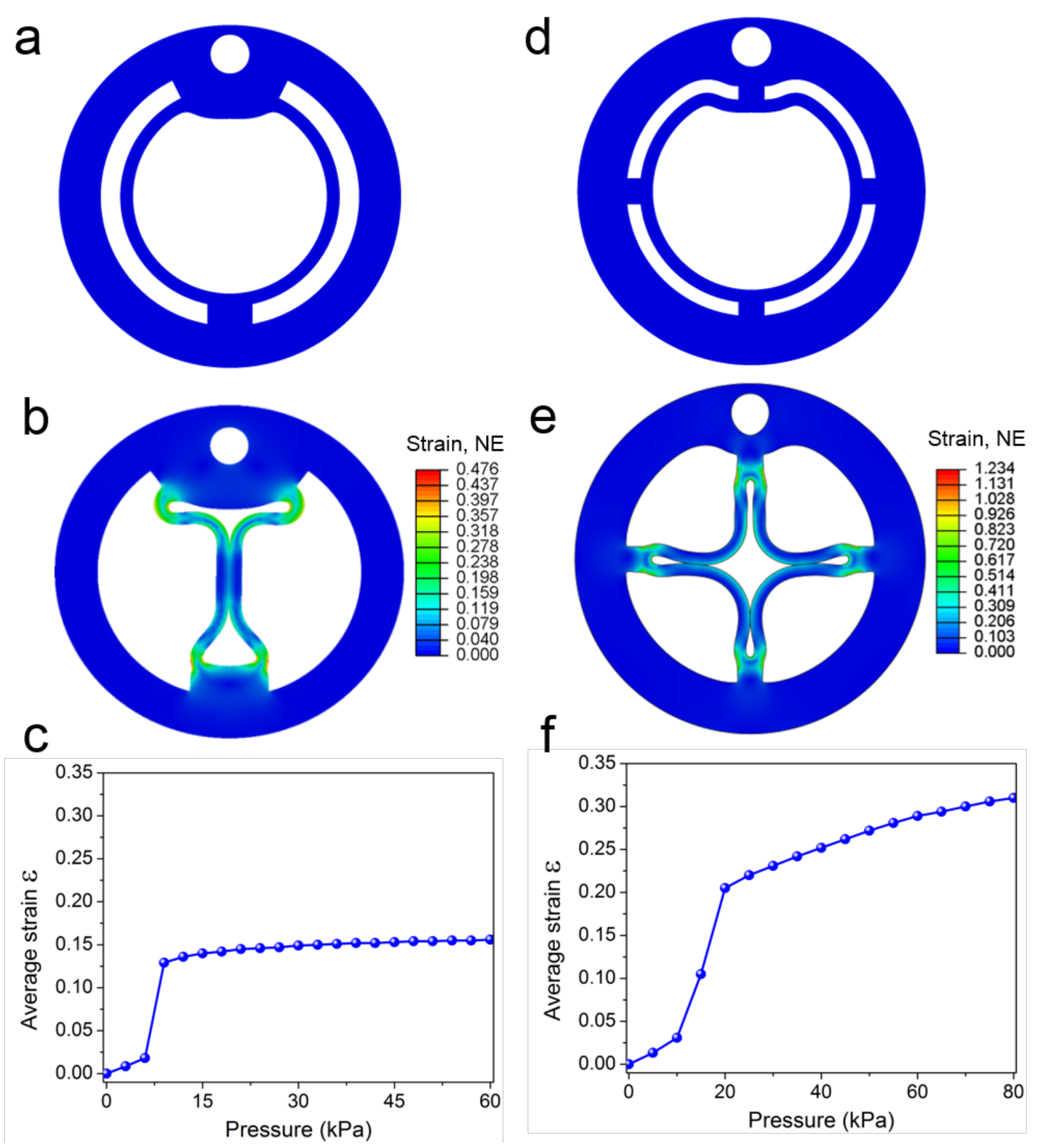

Fig. 2: Finite element models show that a four-inflation-lumen design for a urinary catheter shaft achieves higher levels of tensile strains along circumferential direction in the urine luminal surface than a two-inflation-lumen design at the same inflation pressure. (a) Cross-section of catheter shaft with two intra-wall inflation lumens. (b) Predicted strains along circumferential 
direction in the urine luminal surface of the two-lumen catheter from finite element model when both inflation lumens are simultaneously inflated by a pressure of $60 \mathrm{kPa}$. (c) Predicted average strain along circumferential direction in the urine luminal surface as a function of the inflation pressure for the two-inflation-lumen configuration. (d) Cross-section of catheter shaft with four inflation lumens. (e) Predicted strains along circumferential direction in the urine luminal surface of the four-lumen catheter from the finite element model when four inflation lumens are simultaneously inflated by a pressure of $80 \mathrm{kPa}$. (f) Predicted average strain along circumferential direction in the urine luminal surface as a function of the inflation pressure for the four-lumen configuration. 

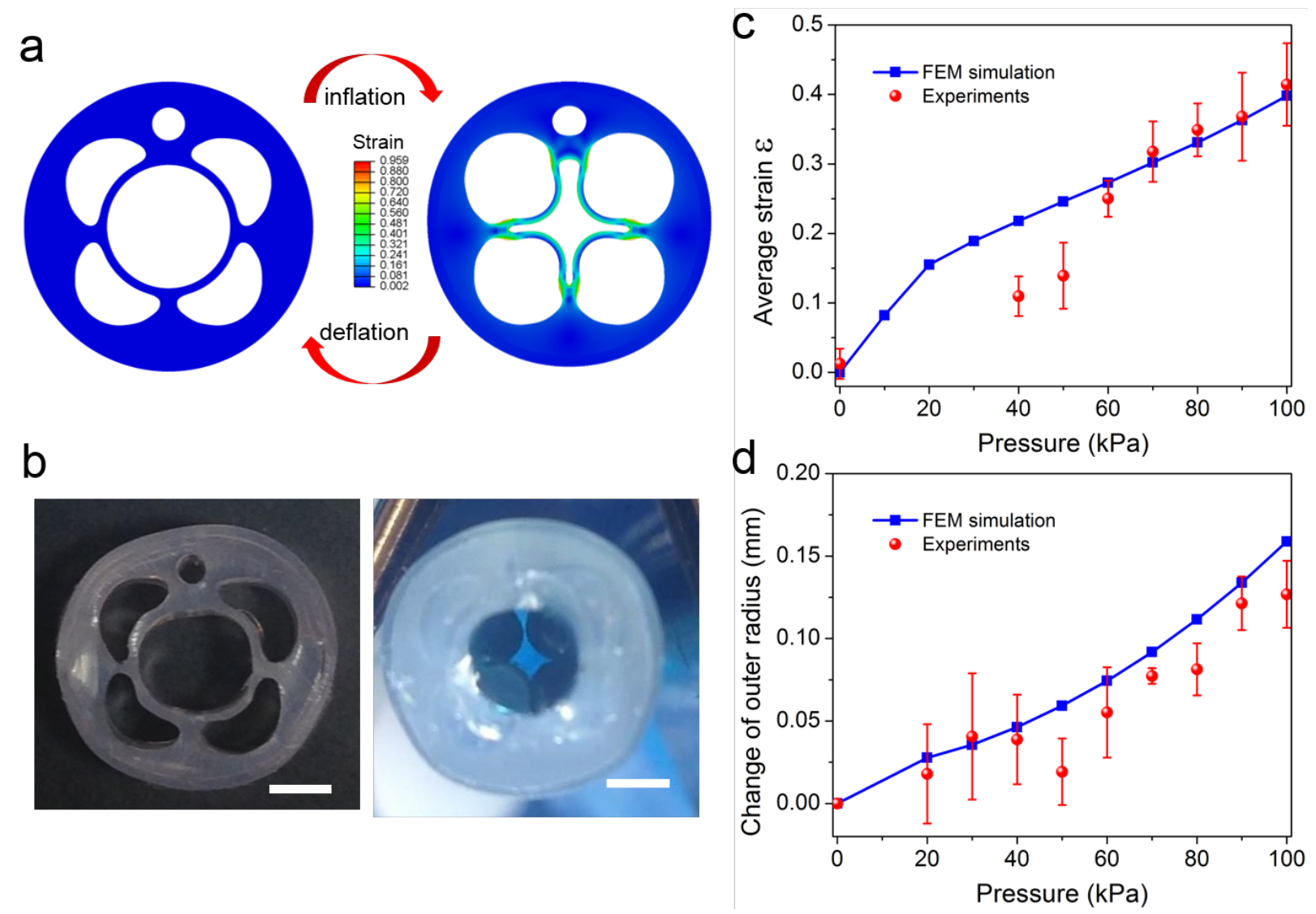

Fig. 3. Experimental testing of a catheter shaft agrees well with the numerical prediction of strain in the central luminal surface as a function of inflation pressure. (a) Cross-section and finite element model for a silicone urinary catheter shaft with four inflation lumens. Strain contour plot from finite element model subjected to an inflation pressure of $80 \mathrm{kPa}$. (b) Digital photograph of the cross-section of a catheter shaft made of 35 durometer, low modulus silicone and constrained with a 65 durometer, high-modulus silicone sheath; and a representative image of its profile when inflated to $80 \mathrm{kPa}$. Scale bar indicates $1 \mathrm{~mm}$. (c) Calculated and experimental average strains along circumferential direction in the central luminal surface. (d) The increase in the outer radius of the shaft as a function of applied inflation pressure. 

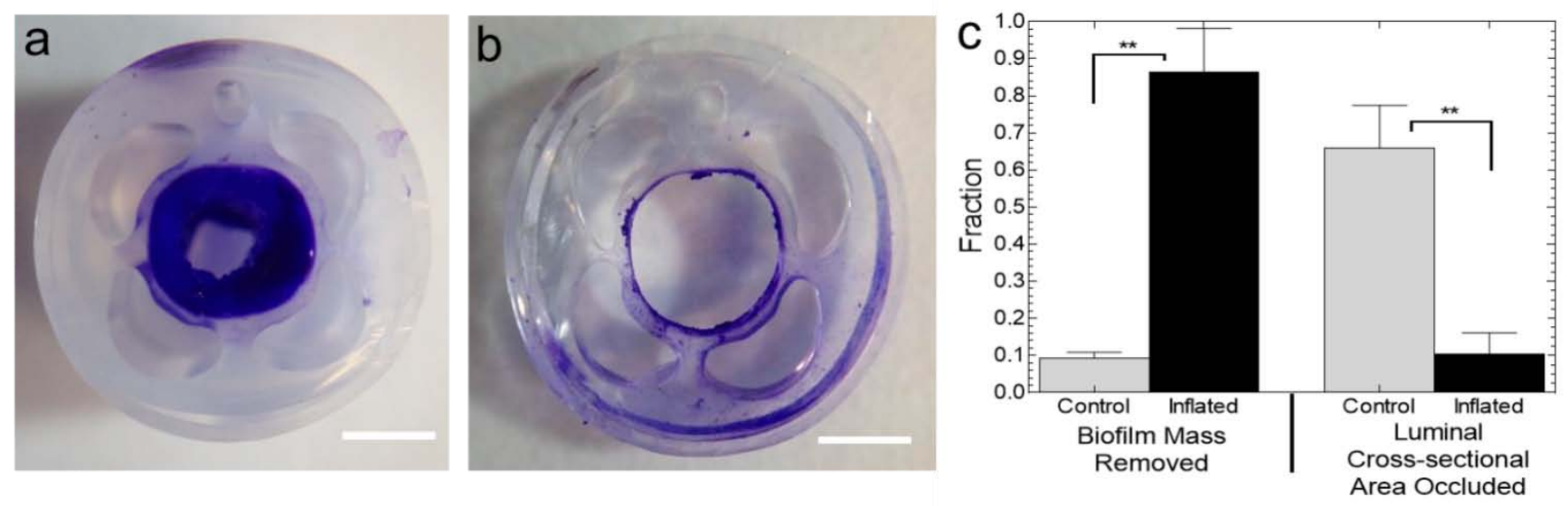

Fig. 4. Prototype fouling-release urinary catheter debonds mixed community $P$. mirabilis and $E$. coli biofilm. Representative optical images of the cross sections of urinary catheter shafts with biofilm intact on the main lumen of (a) control (uninflated) prototypes and debonded from (b) inflated prototypes. All catheters were rinsed at $4 \mathrm{~mL} \mathrm{~min}{ }^{-1}$ with artificial urine for 1 minute. Catheters designated for inflation were inflated to $80 \mathrm{kPa}$ (approximately $35 \%$ strain) 10 times. Sections of the catheter shaft were removed and stained with crystal violet to enhance biofilm visualization. Scale bars in panels (a) and (b) indicate $1 \mathrm{~mm}$. (c) Inflation removed a significant fraction of $P$. mirabilis and $E$. coli biofilm mass as determined by weight of biofilm in the rinse effluent normalized to the weight of biofilm grown in each run. Similarly, biofilm occluded a large fraction of control samples' luminal cross-sectional area, but was removed from inflated samples. $\mathrm{N}=3$ replicates, “**” indicates $\mathrm{p}<0.01$. 

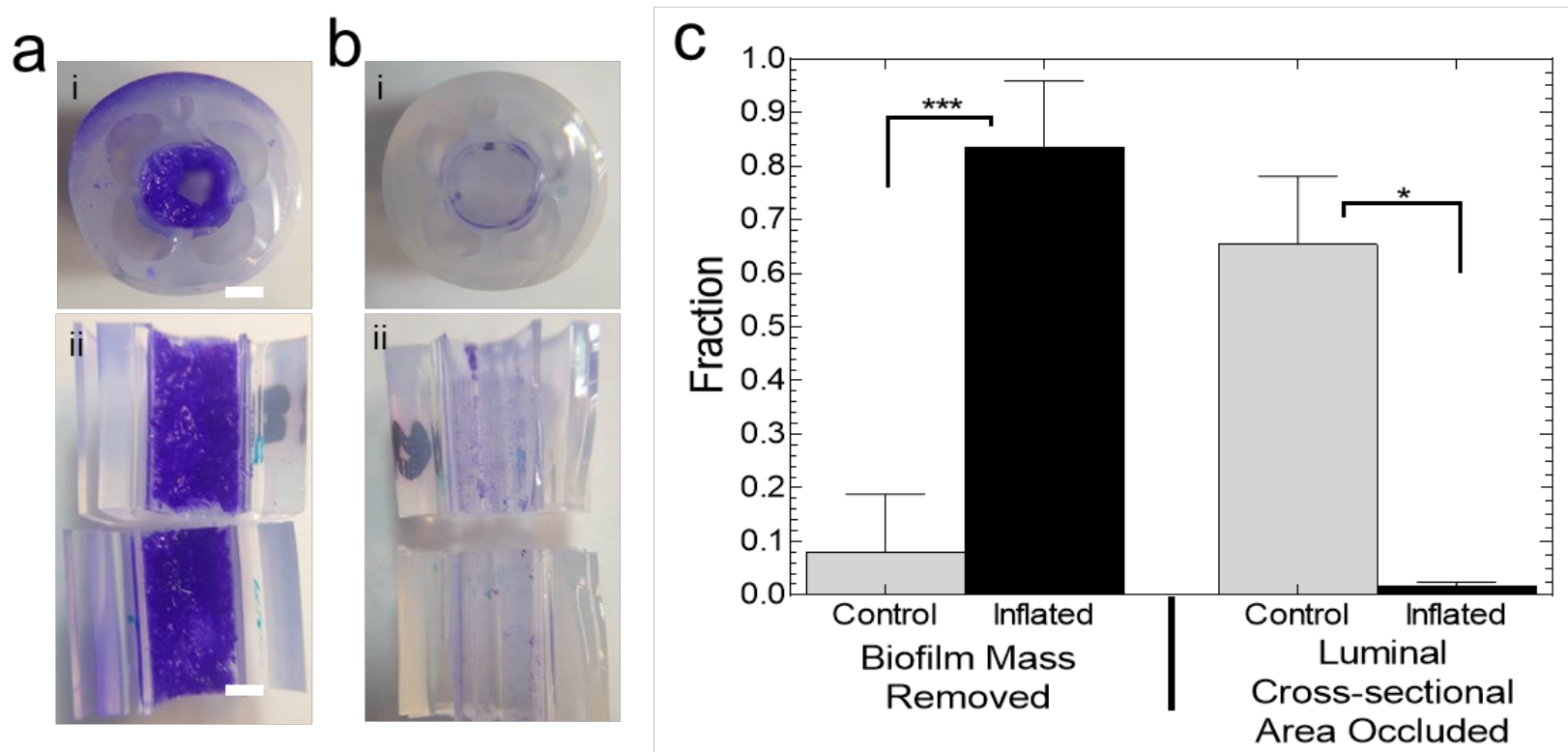

Fig. 5. Prototype urinary catheter repeatedly debonded biofilms with mixed communities of $P$. mirabilis and E. coli. Biofilms were re-grown on samples that had undergone actuation. Samples were rinsed at $4 \mathrm{~mL} \mathrm{~min}^{-1}$ of artificial urine for 1 minute. Catheters designated for inflation were inflated to $100 \mathrm{kPa}$ (approximately $40 \%$ average strain) 10 times. Sections of the catheter shaft were removed and imaged, and select sections were crystal violet stained to enhance biofilm visualization. (a) Representative optical images from control samples (no inflation); both (i) cross section and (ii) sliced open samples show thorough biofilm coverage. Scale bar indicates $1 \mathrm{~mm}$. (b) Representative optical images from inflated samples; (i) both cross section and (ii) sliced open samples show substantial biofilm removal. (c) Inflation removed a significant fraction of re-grown $P$. mirabilis and E. coli biofilm mass as determined by weight of biofilm in the rinse effluent normalized to the weight of biofilm grown in each run $(\mathrm{N}=4$ replicates). Likewise, biofilm occluded a large fraction of control samples' luminal cross- 
sectional area, but was removed from inflated samples ( $\mathrm{N}=3$ replicates). “***” indicates $\mathrm{p}<0.001$ and “*” denotes $\mathrm{p}<0.05$. 

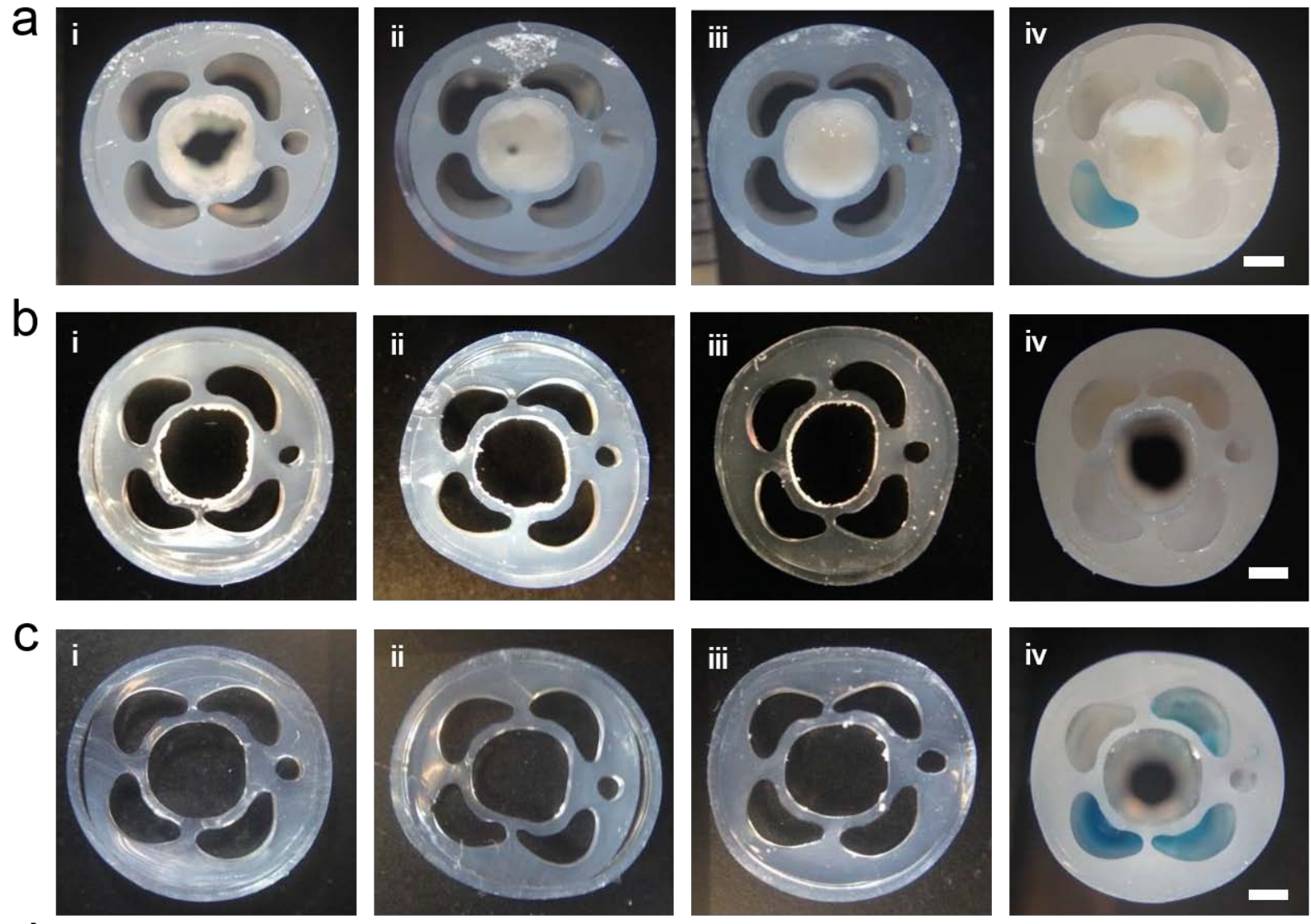

d

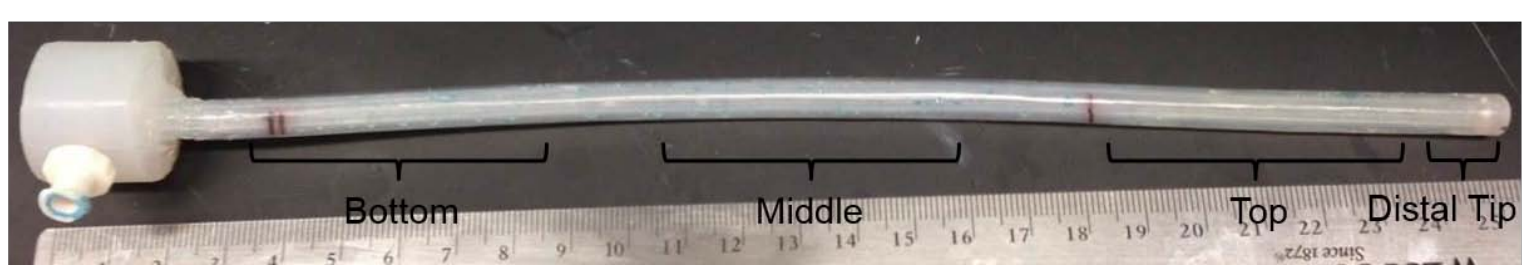

Fig. 6. Prototype urinary catheters debond biofilms with mixed communities of $P$. mirabilis and E. coli along the full length of the catheter shaft. Representative optical images of the cross sections from (a) control catheter (no inflation), (b) first round of inflation after $30 \mathrm{~h}$ of growth of biofilm, and (c) second round of debonding after re-growing the biofilm for another $24 \mathrm{~h}$. (d) Sections were taken from the prototypes at the following locations: (i) bottom, (ii) middle, (iii) top, and (iv) distal tip. Blue coloring in intra-wall inflation lumens is an artifact of residual colored water used for inflation. Scale bars indicate $1 \mathrm{~mm}$. 
a

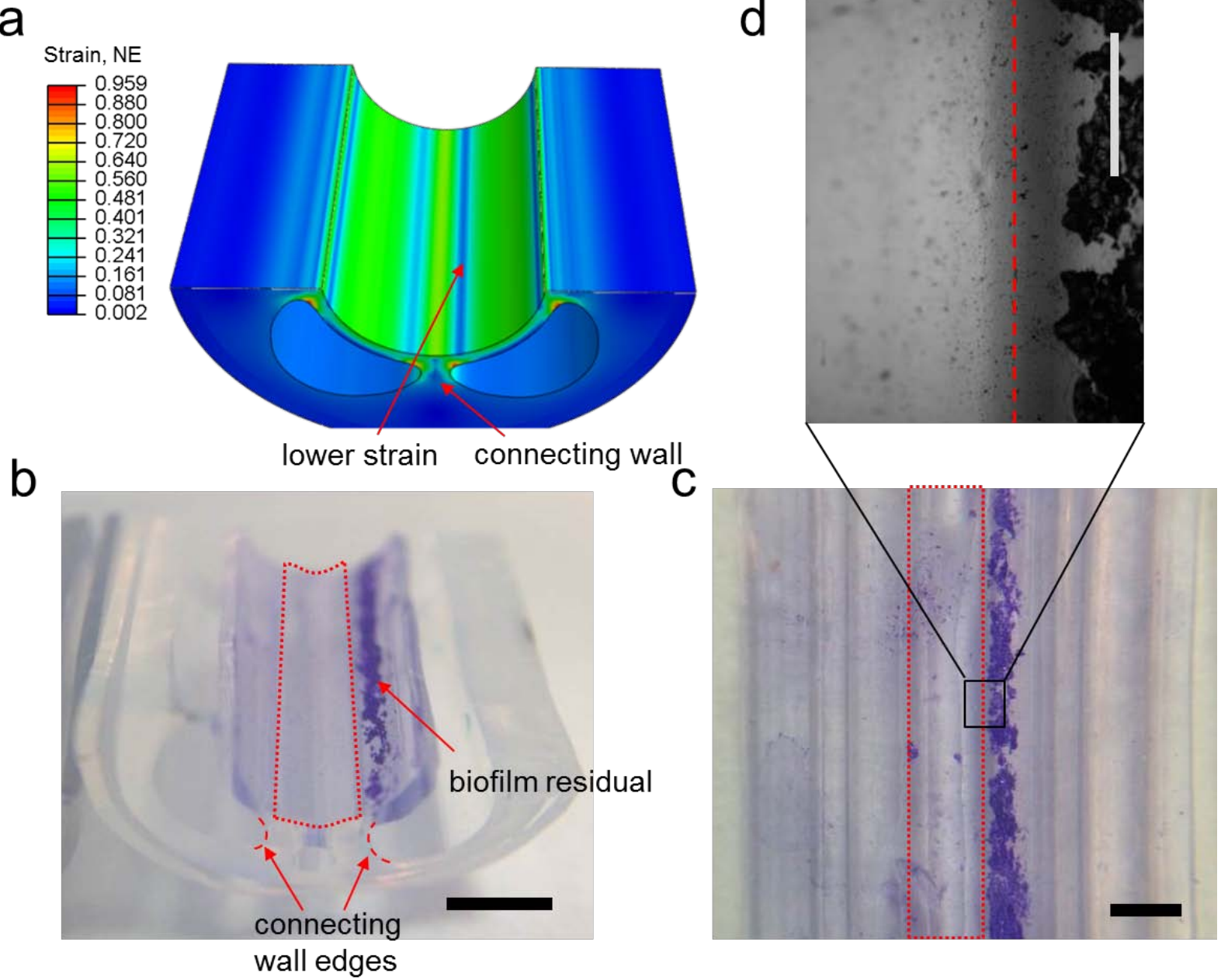

Fig. 7. Compressive strain along luminal surface also debonds biofilm. (a) Contour plot of nominal strains (absolute values of compressive and tensile strains) along luminal surface during inflation mapped onto undeformed surface calculated from the finite element model. Compressive strain is generated in the luminal surface over the connecting walls due to the compression from adjacent inflation lumens during inflation. Lower absolute values of strain appear at the edge of inflation lumens due to the transition from tensile to compressive strain in that region. (b) Optical image of sliced-open crystal violet stained section of a catheter shaft that experienced two rounds of biofilm growth and debonding. Red, dashed box highlights the region on the luminal surface that has substantial biofilm removal due to compressive strain. (c) Optical 
image of luminal surface excised from catheter and flattened. Scale bars in panels (b) and (c) indicate $1 \mathrm{~mm}$. (d) Optical microscopic image of luminal surface overlying the boundary between the wall and the inflation lumen. Dashed line shows area overlying connection wall. Biofilm is visible at edge of inflation area, where low levels of strain were predicted by the finite element model calculations. Scale bar in panel (d) indicates $500 \mu \mathrm{m}$. 